\title{
Silicon-Based Micro-Fourier Spectrometer
}

\author{
Dietmar Knipp, Member, IEEE, Helmut Stiebig, Sameer R. Bhalotra, Student Member, IEEE, Eerke Bunte, \\ Helen L. Kung, Student Member, IEEE, and David A. B. Miller, Fellow, IEEE
}

\begin{abstract}
A novel Fourier spectrometer based on a partly transparent thin-film detector in combination with a tunable silicon micromachined mirror was developed. The operation principle based on the detection of an intensity profile of a standing-wave by introducing a partly transparent detector in the standing-wave. Varying the position of the mirror results in a phase shift of the standing-wave and thus in a change of the optical intensity profile within the detector. The photoelectric active region of the sensor is thinner than the wavelength of the incoming light, so that the modulation of the intensity leads to the modulation of the photocurrent. The spectral information of the incoming light can be determined by the Fourier transform of the sensor signal. Based on the linear arrangement of the sensor and the mirror, the spectrometer facilitates the realization of one- and two-dimensional arrays of spectrometers combining spectral and spatial resolution. The operation principle of the spectrometer will be described and the influence of the detector design on the spectrometer performance will be discussed. A spectral resolution of down to $6 \mathrm{~nm}$ was achieved under real-time imaging conditions.
\end{abstract}

Index Terms-Fourier spectrometer, hyperspectral imaging, microelectromechanical-systems (MEMS), micromachining, multispectral imaging, optical sensor, spectrometer, thin films.

\section{INTRODUCTION}

$\mathbf{V}$ ARIOUS microspectrometers for different applications have been developed in recent years [1]-[6]. Especially the progress in optical microelectromechanical systems (MEMS) technology has initiated a lot of new research activities in the field of the optical metrology. The applications of microspectrometers range from chemical and biological analysis to inspection and multispectral imaging. The activities are driven by improvements of size, cost and performance. Consequently, several spectrometer concepts were transferred from the macro to the microscale like grating spectrometers [1], [2], Fabry-Perot spectrometers [1]-[3] and Fourier spectrometers [4]-[6].

Manuscript received July 28, 2004; revised November 29, 2004. This work was supported in part by the "Deutsche Forschungsgemeinschaft" (JA779/4), Sti $181 / 1-1$ and in part by DARPA under a Grant for Photonic Wavelength and Spatial Signal Processing. S. R. Bhalotra gratefully acknowledges the Regina Casper Stanford Graduate Fellowship. The review of this paper was arranged by Editor K. Najafi.

D. Knipp is with the Department of Science and Engineering, International University Bremen, 28759 Bremen, Germany.

H. Stiebig and E. Bunte are with the Research Center Jülich, Institute of Photovoltaic, 52425 Jülich, Germany.

S. R. Bhalotra and D. A. B. Miller are with the Ginzton and Solid State and Photonics Laboratories, Stanford University, Stanford, CA 94305 USA.

H.L. Kung was with the Ginzton and Solid State and Photonics Laboratories, Stanford University, Stanford, CA 94305, USA. She is now with California Technology and Manufacturing, Intel Corporation, Santa Clara, CA 95054 USA.

Digital Object Identifier 10.1109/TED.2005.843880
For several applications like inspection or multispectral imaging it is highly desired to acquire the spectral and the spatial information of the object at the same time, which requires the integration of several hundreds or even thousands of spectrometers on a sensor chip. Grating spectrometers and classical Fourier spectrometer do not allow for the dense integration of spectrometers on a single chip. Fabry-Perot spectrometers can be densely integrated on a sensor chip. However, the resolution of the spectrometer is determined by the finesse of the spectrometer, which is limited by the reflectivity of the semi transparent mirrors.

In the following paper, we will present a new concept of a spectrometer, which allows for the densely integration of spectrometers on a sensor chip. The spectral information is generated by the sampling of a standing-wave. The key component of this novel spectrometer is a partly transparent optical detector. The partly transparent sensor is introduced in a standing-wave created in front of a tunable mirror. The detector is very thin so that the sensor can sample the intensity profile of the standing-wave. The spectrometer operates in the visible part of the optical spectrum so that it can be used for several applications.

The concept of standing-wave based wavelength sensitive detectors [7], interferometers and spectrometers is known for many years [8]-[10], [21], [22], but the realization of such concepts was limited by technological reasons. Sampling of a standing-wave by a semiconductor device requires 1) a highly transparent, and 2) a very thin detector with 3) an adequate photosensitivity which is realized on a transparent substrate.

The high transmittance of the detector is a prerequisite to create a standing-wave in front of the mirror. On the other hand sufficient light has to be absorbed to generate an electrical signal by the sensor. The photocurrent will be modulated as a consequence of the optical intensity profile. The active region of the sensor should be thinner than the wavelength of the incoming light within the material so that the photocurrent is modulated by the displacement of the mirror. A silicon based partly transparent detector operating in the visible part of the spectrum can be realized by using absorber thicknesses of 30-50 nm [10]-[12]. In the case of a thicker active layer the photocurrent is still modulated but the amplitude of the modulated signal is reduced.

Kung and coworkers [10], [11] presented the first Fourier spectrometer utilizing the effect of sampling a standing-wave in the visible part of the spectrum. In this case a thin amorphous silicon coplanar photoconductor structure was applied to sample a standing-wave created in front of a micromachined mirror. The same concept can be extended to the infrared part of the spectrum by using a partly transparent GaAs photodiode [12].

In both cases, the novel spectrometer concept reduces the number of components to a minimum, simplifies the set-up to a 
linear arrangement of a detector and a mirror and facilitates the realization of one-dimensional (1-D) or two-dimensional (2-D) spectrometer arrays.

The performance of the spectrometer is mainly determined by the accuracy of the thin film processing of the partly transparent sensor, the optoelectronic properties of the active thin films, the interaction of the standing-wave with the detector, and the performance of the micromachined mirror. In this paper we will mainly focus on the description and characterization of the partly transparent sensors. A partly transparent amorphous silicon photodiode was used to realize the micro-fourier spectrometer. In Section II of this paper we present the operation principle of a standing-wave spectrometer. The optical properties of the spectrometer will be discussed in Section III. A detailed description of the experimental results will be given in Section IV. The implications of partly transparent sensor and the micromachined mirror with respect to the performance of the spectrometer are discussed in Section V.

\section{FUNDAMENTALS}

\section{A. Standing-Wave}

The interference of two waves can be described by the superposition of two optical beams propagating in the same or opposite direction. The amplitude and phase of the electric field determine the intensity at each spatial position of the interference pattern. The intensity of the superimposed waves can be described by (1)

$$
I_{S}(x)=I_{1}(x)+I_{2}(x)+2 \cdot \sqrt{I_{1}(x) I_{2}(x)} \cos \left(\frac{4 \pi \cdot d_{m}}{\lambda}\right) .
$$

$I_{1}(x)$ and $I_{2}(x)$ are the intensities of the interfering beams and $4 \pi \cdot d_{m} / \lambda$ is the phase difference of the beams. In the case of a Michelson interferometer the standing-wave in the detector arm is created by the superposition of two waves propagating into the same direction and $d_{m}$ corresponds to the relative displacement of the reference and measuring mirror. If the intensity of the two beams is equal, $I_{1}(x)=I_{2}(x)=I_{0},(1)$ is simplified to

$$
I_{S}=2 I_{0}\left(1+\cos \left(\frac{4 \pi \cdot d_{m}}{\lambda}\right)\right)=4 I_{0} \cos ^{2}\left(\frac{2 \pi \cdot d_{m}}{\lambda}\right) \text {. }
$$

As a consequence the signal varies between 0 (destructive interference) and $4 \cdot I_{0}$ (constructive interference).

\section{B. Standing-Wave spectrometer}

In the case of a standing-wave spectrometer the two superimposed waves propagate in opposite directions. The working principle of an ideal standing-wave spectrometer is schematically depicted in Fig. 1.

The reflection of the incoming light intensity $I_{0}$ at the mirror leads to the creation of a standing-wave in front of the mirror. By moving the detector or reflector, the optical intensity profile within the detector changes. The intensity profile can be described according to (1). The wavelength of the standing-wave corresponds to twice of the wavelength of the incoming light. Due to the absorption of a fraction of the incoming light within the detector and reflection losses at the detector and at the mirror the light intensity $I_{2}$ is always smaller than the incident light

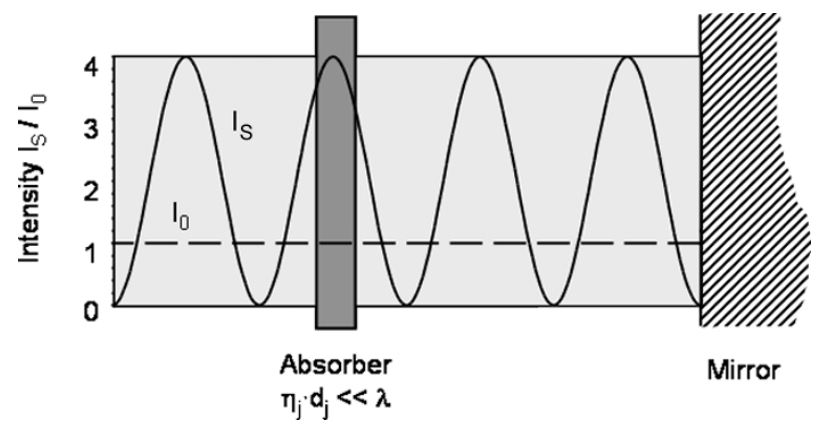

Fig. 1. Schematic setup of a standing-wave spectrometer consisting of a party transparent optoelectronic active absorber and a mirror.

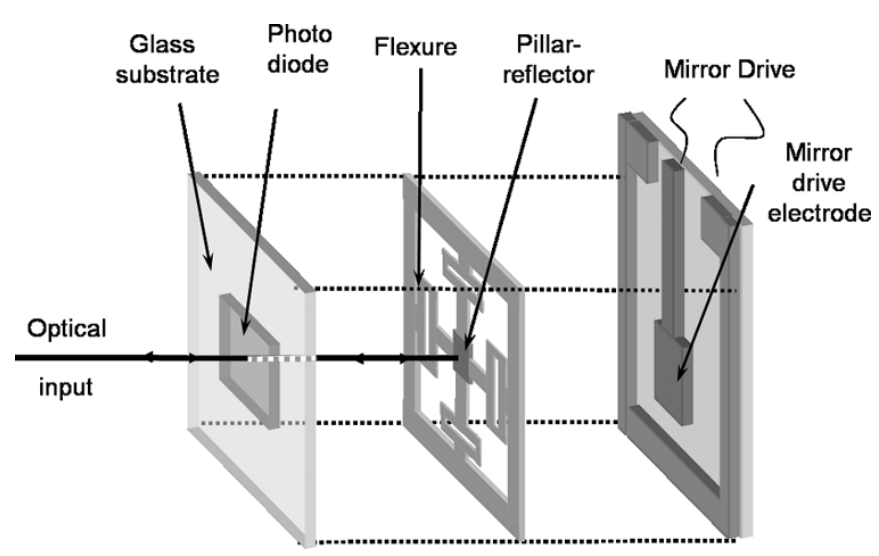

Fig. 2. Schematic sketch of a Fourier spectrometer based on a partly transparent sensor on a glass substrate and a micromachined mirror.

intensity $I_{1}\left(I_{1}(x)>I_{2}(x)\right)$. Therefore, the ideal case of complete constructive or destructive interference, which is displayed in Fig. 1, is not reached.

A schematic sketch of a standing-wave spectrometer is shown in Fig. 2. The spectrometer consists of the partly transparent sensor on a glass substrate and a micromachined, electrostatically tunable mirror. An actuator frame, which holds the reflector and a second driver electrode realized on a subsequent wafer, forms the MEMS mirror. The fabrication and the properties of the tunable mirror are described in [10]-[12].

Different partly transparent detector structures can be used to sample the standing-wave like coplanar photoconductors or photodiodes. In the following we will focus on nip-photodiodes based on amorphous silicon (Fig. 3). The amorphous silicon diode is embedded between two transparent conductive oxide layers, which form the front and the back electrode of the sensor.

The application of a nip-photodiode has several advantages in terms of the optical design of the sensor. The individual regions of the photodiode can be designed nearly independent of each other like the absorption region, the entire amorphous silicon based layer thickness of diode and the transparent conductive front and back contacts, which act as the anti-reflex coating of the amorphous layer system [13].

\section{OPTICAL MODEL}

In order to describe the wave propagation within the partly transparent sensor, an optical model based on the Airy formalism [14] was used. The performance of the optical system 


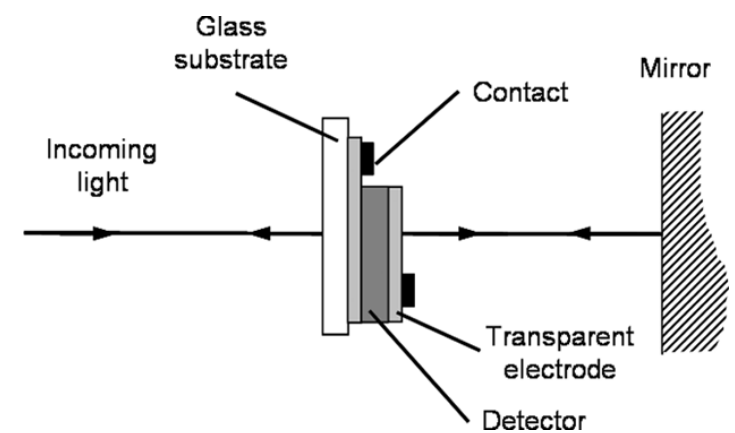

Fig. 3. Schematic sketch of the semi transparent sensor of a standing-wave Fourier spectrometer. Light reflected by the mirror creates a standing-wave in front of the mirror, which is detected by the partly transparent detector. The partly transparent sensor is formed by a sandwich structure.

can be described by a linear function, which directly correlates the input and the output electric field vector. The details of the optical model were described elsewhere [15], [16]. Fig. 4 exhibits a universal layer structure, which consists of a partly transparent detector, an air gap and a mirror.

The component $E_{0,1}^{+}$describes the electric field toward the first interface within positive direction, whereas $E_{0,1}^{-}$represents the reflected electric field (field propagation in negative direction) of the whole structure and it is defined at the interface between layer 0 and 1 . The component $E_{m+1, m}^{+}$describes the electric field at the interface between the $m$ - and $m+1$-layer and considers the part of the light, which is coupled in the mirror (layer $m+1$ ). Since no light enters the optical system from the backside, $E_{m+1, m}^{-}$becomes zero. Layer $j$ denotes the optoelectronic active layer of the detector and the layer $m$ corresponds to the air gap between the detector and the mirror. In order to simplify the description of the optical wave propagation, the layer stack can be divided into a four-layer system based on the $j$-layer (active region of the sensor), the two subsystems $S_{1}$ (layer 1 to $j-1$ ) and $S_{2}$ (layer $j+1$ to $m-1$ ) and the m-layer (air gap between the mirror and the detector). At this point we assume that the mirror has a reflectivity of $100 \%\left(E_{m+1, m}^{-}=0\right)$. Based on this expression the electric field in the $j$-layer can be calculated as a superposition of the electric field in positive and negative directions. Subsequently the intensity within the layer $j$ (3) can be calculated after a few transformations [13], [14]

$$
\begin{aligned}
I_{j}\left(x, d_{m}, \lambda\right)= & I_{0} \mathrm{~T}_{0}(\lambda) \frac{\eta_{j}}{\eta_{0}}\left(\mathrm{e}^{-\alpha_{j} x}+\left(\tau_{2}\right)^{2} \cdot \mathrm{e}^{-\alpha_{j}\left(2 d_{j}-x\right)}\right. \\
& -2 \cdot \tau_{2} \cdot \mathrm{e}^{-\alpha_{j} d_{j}} \\
& \left.\cdot \cos \left(\frac{4 \pi}{\lambda}\left[\eta_{j}\left(d_{j}-x\right)+\eta_{m} d_{m}\right]+2 \xi_{2}\right)\right)
\end{aligned}
$$

where $I_{0}$ is the incident light intensity and $T_{0}(\lambda)$ corresponds to the internal transmission of the multilayer system, $\alpha_{j}$ is the absorption coefficient of the $j$-layer $\left(\alpha_{j}=4 \pi \kappa_{j} / \lambda\right), \eta_{j}$ and $\eta_{0}$ are the real components of the complex refractive indices $(n=\eta+i \kappa)$ of the $j$-layer and the ambient layer (typically air), respectively, and $\tau_{2}$ and $\xi_{2}$ are the absolute value and the phase of the transmittance of the subsystem $S_{2}$. The first term $\exp \left(-\alpha_{j} x\right)$ of (3) originates from the electric field propagation in positive direction. The positive direction means the direction of the incident light. The second term $\exp \left(-\alpha_{j}\left(d_{j}-x\right)\right)$ describes the intensity, which propagates in negative direction. The third term is caused by interference effects of the two waves.

In order to design the transparent detector the following aspects have to be considered. The sensor has to be highly transparent so that a standing-wave can be formed in front of the mirror. On one hand a sufficient fraction of the light has to be absorbed to generate a photocurrent. However, it has to be distinguished between the overall photocurrent and the photocurrent generated as a consequence of the modulated standing-wave. The overall photocurrent corresponds to the dc sensitivity (SEN) of the sensor, whereas the current generated by the standingwave is represented by the differential sensitivity (DS) of the sensor. As a quantity for the signal to noise ratio the selectivity (SEL) is used.

We calculated the dc SEN by an integration of the intensity over the active layer ( $j$-layer). For simplicity we assumed that the transmission of the layer stack $S_{2}$ is equal to $1\left(\tau_{2}=1\right)$ and the phase information of the subsystem $S_{2}$ is equal to 0 $\left(\xi_{2}=0\right)$. Further, we neglected the influence of the interference effect on the sensitivity. After integration the sensitivity is given by (4)

$$
\begin{aligned}
\operatorname{SEN}(\lambda) & =I_{0} \frac{\eta_{j}}{\eta_{0}} \int_{0}^{d_{j}}\left(\exp \left(-\alpha_{j} x\right)+\exp \left(-\alpha_{j}\left(2 d_{j}-x\right)\right) \mathrm{d} x\right. \\
& =\frac{\mathrm{k}_{0}}{\alpha_{j}}\left(1-\exp \left(-2 \alpha_{j} d_{j}\right)\right) .
\end{aligned}
$$

The constant $k_{0}$ is equal to $I_{0} \cdot \eta_{j} / \eta_{0}$. In Fig. 5(a) the sensitivity is plotted as a function of the thickness of the active layer.

We used an amorphous silicon layer for the calculations assuming the wavelength of the incoming light to $550 \mathrm{~nm}$ (green). The sensitivity increased exponentially with thickness until nearly all light is absorbed $\left(d_{j}=200-300 \mathrm{~nm}\right)$ and the sensitivity remains constant. The same parameters were used for the calculations of Fig. 5(b) and (c). Besides the dc sensitivity, we are mainly interested in the interdependency of the standing-wave with the partly transparent sensor. Therefore, we calculated the DS of the spectrometer corresponding to the interference term in (3)

$$
\begin{aligned}
\operatorname{DS}\left(d_{m}, \lambda\right)= & -\mathrm{k}_{0} \exp \left(-\alpha_{j} d_{j}\right) \\
& \times \int_{0}^{d_{j}} \cos \left(\frac{4 \pi}{\lambda}\left(\eta_{j}\left(d_{j}-x\right)+d_{m}\right)\right) d x \\
= & \frac{k_{0} \lambda}{\pi \eta_{j}} \exp \left(-\alpha_{j} d_{j}\right) \cos \left(\frac{4 \pi d_{m}}{\lambda}+\frac{2 \pi \eta_{j} d_{j}}{\lambda}\right) \\
& \cdot \sin \left(\frac{2 \pi \eta_{j} d_{j}}{\lambda}\right)
\end{aligned}
$$

The DS as a function of the $j$-layer thickness is shown in Fig. 5(b). It is a measure for the alternating component of the photocurrent, which is used for the deconvolution. We calculated the DS (thin solid lines) for two different mirror positions $\left(d_{m}=\lambda / 4\right.$ and $\left.d_{m}=\lambda / 2\right)$. The two mirror positions are relatively spaced by $d_{m}=\lambda / 4$, which corresponds to a phase shift of $90^{\circ}$. The bold solid lines in Fig. 5(b) correspond to the upper and lower limit of the DS. For thin active layers we observe an 


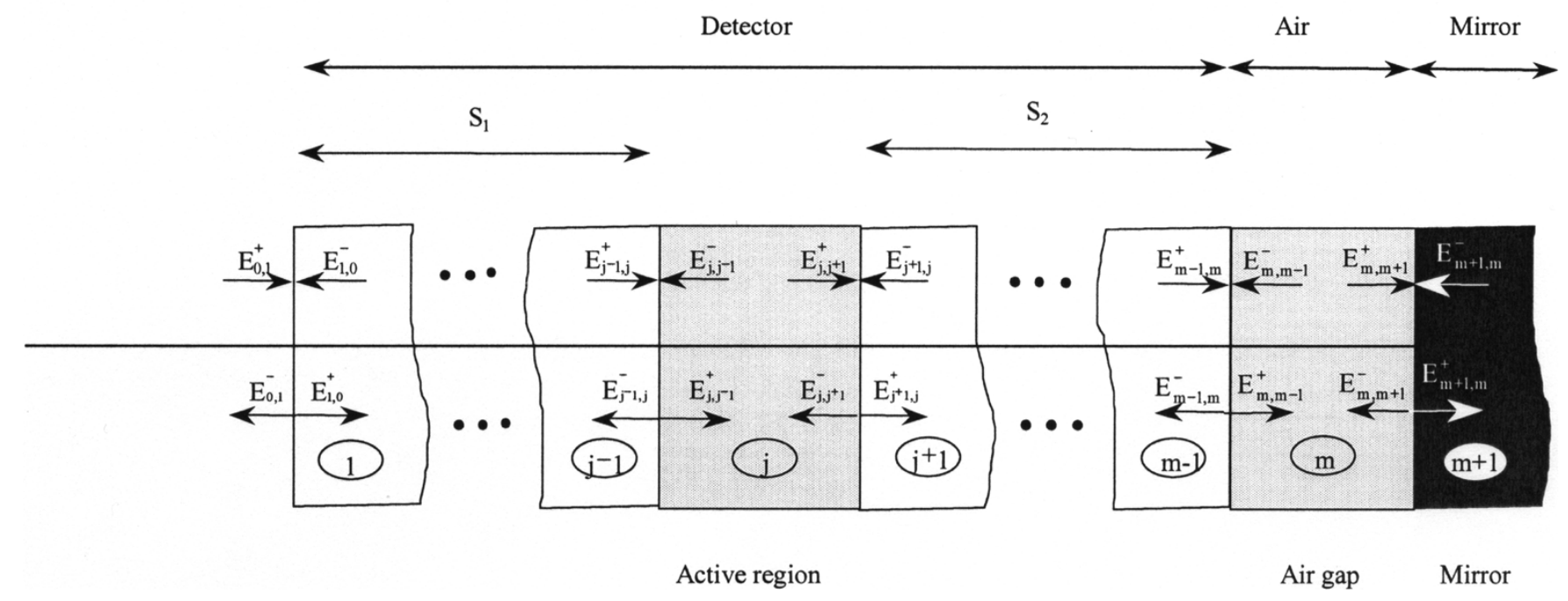

Fig. 4. Schematic sketch of a spectrometer based on a multilayer system. The layer $j$ represents the optoelectronic active layer of the detector and the layer $\mathrm{m}$ corresponds to the air gap between the mirror and the detector. The system consists of the subsystem $S_{1}$, the optoelectronic active layer $j$, the subsystem $S_{2}$, the air gap layer $m$ and the mirror (layer $m+1$ ).

increase of the DS with increasing thickness of the active layer. In this case, the active layer is distinctly thinner than the wavelength $\left(d_{j} \ll \lambda / \eta_{j}\right)$ and the increase of the DS is caused by the increased absorption of the active layer. The DS reaches its maximum for an active layer thickness of $30 \mathrm{~nm}$. For thicker active layers the DS decreases again. Less light is reflected at the mirror, which contributed to the standing-wave and therefore to the DS. The nodes at a multiple of $\lambda /\left(2 n_{j}\right)$ indicates that the amplitude of the alternating photocurrent is zero when the $j$-layer thickness of the device equals the period or a multiple of the period of the standing-wave.

A further figure of merit is the SEL. We defined the SEL as the ratio of the DS and the dc sensitivity. The SEL can be described by the combination of (4) and (5) resulting in (6). It describes the ratio of the alternating and the direct component of the photocurrent.

Again, we neglected the effect of the subsystems $S_{2}\left(\tau_{2}=1\right)$ on the standing-wave. Therefore, the SEL depends only on the material properties of the active layer, the wavelength of the incident light and the air gap between the sensor and the mirror. The SEL as a function of the thickness of the active film is shown in Fig. 5(c)

$$
\begin{aligned}
& \operatorname{SEL}\left(d_{m}, \lambda\right) \\
& =\frac{\operatorname{DS}\left(d_{m}, \lambda\right)}{\operatorname{SEN}(\lambda)} \\
& =\frac{\exp \left(-\alpha_{j} d_{j}\right) \cos \left(\frac{4 \pi d_{m}}{\lambda}+\frac{2 \pi \eta_{j} d_{j}}{\lambda}\right) \sin \left(\frac{2 \pi \eta_{j} d_{j}}{\lambda}\right)}{1-\exp \left(-2 \alpha_{j} d_{j}\right)} .
\end{aligned}
$$

The curves were again determined for the two mirror positions of $d_{m}=\lambda / 4$ and $d_{m}=\lambda / 2$ (thin solid lines). The bold solid lines correspond to the upper and lower limit of the SEL. For thin active layers most of the light is transmitted through the sensor and a strong modulation is detected. In this case the active layer is thinner than the period of the standing-wave. The $\mathrm{SEL}$ reaches its maximum at $\operatorname{SEL}\left(d_{j} \ll \frac{\lambda}{\eta_{j}}\right)= \pm 1$. The SEL decreases for an $i$-layer thickness thicker than $10 \mathrm{~nm}$. With increasing thickness the modulation decreases, because the transmission of the sensor is reduced.

The calculations in Fig. 5(a)-(c) clearly illustrates the relation between the dc sensitivity, the DS, the active layer thickness and the position of the mirror. To realize an optimized partly transparent sensor the DS and the SEL have to be as high as possible. For the investigated single layer system the highest DS and a high SEL is reached for layer thicknesses of 30-50 nm. However, the deduction of an optimized active layer thickness from the calculations can only be used as a rough estimation, because only the active layer of the sensor is considered. The influence of the subsystems $S_{1}$ and $S_{2}$ on the optical generation and the interaction of the whole device with the standing-wave were neglected.

\section{RESULTS}

In the following, we will discuss the experimental results. The partly transparent detectors were realized by nip-photodiodes. The amorphous silicon detectors were deposited in a multichamber plasma enhanced chemical vapor deposition system at $210^{\circ} \mathrm{C}$ on glass substrates coated with flat transparent conductive oxide $\left(\mathrm{TCO}_{I}\right)$ [18]. p- and n-type doped layers were realized by adding trimethylboron and phosphine to the deposition gases silane and hydrogen, respectively [19]. The $i$-layers were prepared by using silane, methane, and hydrogen. A small amount of carbon $(5 \%-10 \%$ volume percent) was alloyed to the silicon to decrease the refractive index of the material slightly. As a consequence the layer thickness can be slightly increased, while keeping the optical thickness of the absorber $\left(d_{j} \cdot \eta_{j}\right)$ 

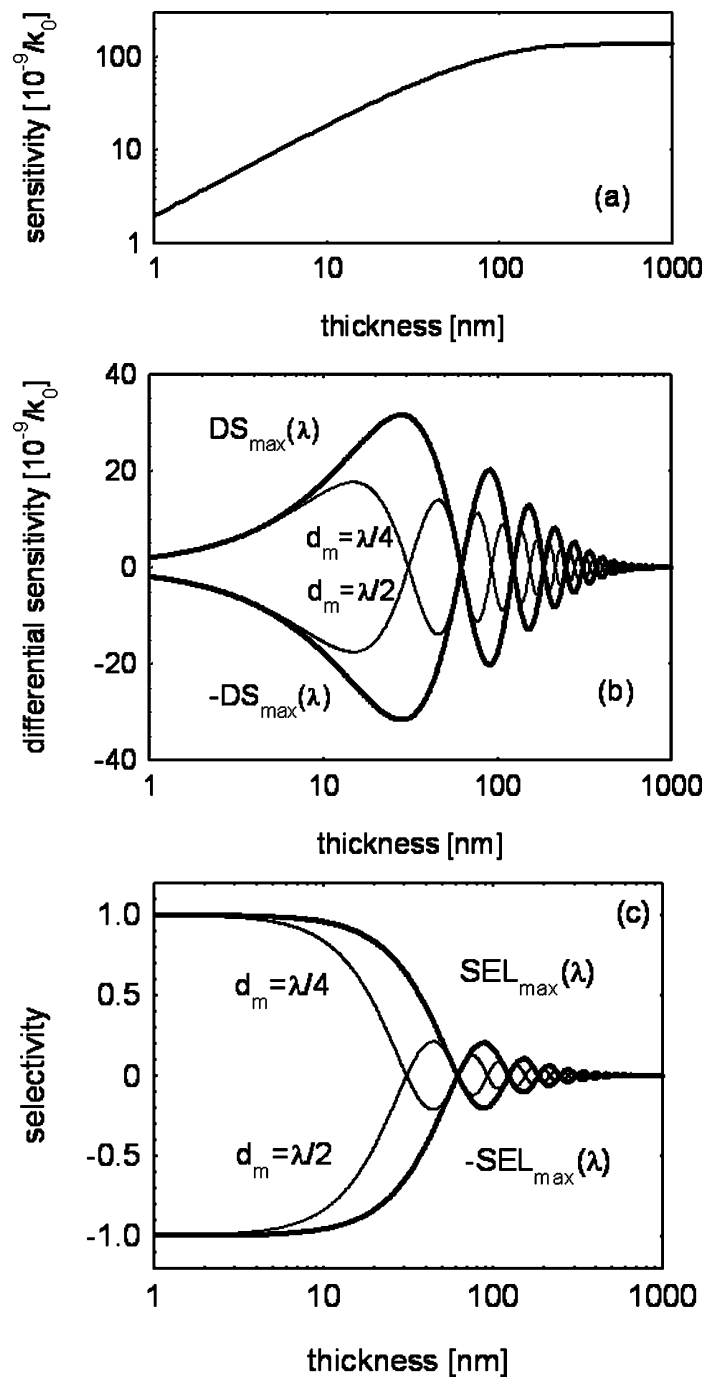

Fig. 5. (a) Sensitivity, (b) DS and (c) SEL of a partly transparent amorphous silicon layer as a function of the thickness of the active layer. We used a wavelength of $550 \mathrm{~nm}$ (green) for the calculations. The amorphous layer is described by the complex refractive index of the material. The thin lines in (a) and (b) correspond to a spacing between the sensor and the mirror of $d_{m}=\frac{\lambda}{4}$ and $d_{m}=\frac{\lambda}{2}$. The bold lines correspond to the lower and upper limits of the DS and SEL.

constant. Moreover, adding carbon leads to a distinct improvement of the device yield. A detailed discussion of the influence of carbon on the amorphous detector is given in [13]. After depositing the amorphous silicon films the second TCO-layer $\left(\mathrm{TCO}_{\text {II }}\right.$ layer) was prepared. The TCO layers were realized by RF-magnetron sputtered $\mathrm{ZnO}$, which is doped with aluminum. To prevent a damage of the amorphous layer system the $\mathrm{TCO}_{\text {II }}$ layer was prepared at room temperature. The multilayer stacks on glass were patterned using photolithography and reactive ion etching. The detectors have an active area of $10 \mathrm{~mm}^{2}$.

The influence of the device design, in particular, the influence of the thickness of the TCO layers and the absorption layer thickness has already been studied by optical calculations and experiments. A detailed description is given in [17]. In this section we concentrate on an optimized device structure (nip-diodes) with TCO-layers corresponding to a multiple of a quarter wavelength $\left(3 * \lambda /\left(4 \eta_{\mathrm{TCO}}\right)\right)$. The device design was

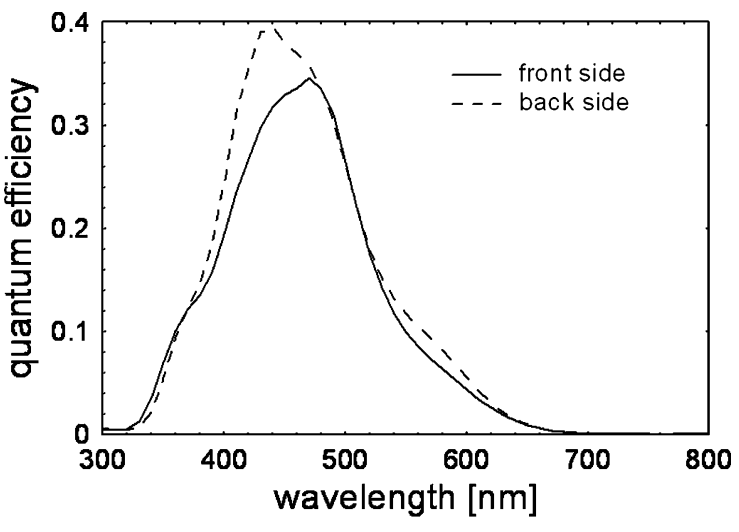

Fig. 6. Measured quantum efficiency of a partly transparent sensor measured from the front and the backside.

optimized for a wavelength of $633 \mathrm{~nm}$, so that the performance can be evaluated using a HeNe laser as a light source.

The amorphous nip-diode has a thickness of $\lambda / 2 \eta_{n},\left(d_{p}=\right.$ $\left.\lambda / 8 \eta_{p}, d_{i}=\lambda / 4 \eta_{i}, d_{n}=\lambda / 8 \eta_{n}\right)$. The $i$-layer thickness of the diode is $40 \mathrm{~nm}$, which is in agreement with the calculations in Fig. 5. The transmission of the partly transparent diode was proven to be $75 \%$ for a wavelength of $633 \mathrm{~nm}$. The quantum efficiency of the detector measured from both sides is shown in Fig. 6.

Similar values are measured independent of the direction of light incident. Thus, the glass substrate has only a minor effect on the measured quantum efficiency. Only the wavelength range between 400 and $500 \mathrm{~nm}$ measured from the $\mathrm{TCO}_{\mathrm{II}}$-side shows a higher sensitivity. The behavior can be likely attributed to the lower absorption losses of the incident light within the wide bandgap p-layer in comparison with the lower bandgap of the n-layer. In order to characterize the spectrometer the thin film sensor was combined with the micromachined mirror. A cross section of the microspectrometer was given in Fig. 2. It is very important for the performance of the spectrometer that the mirror and sensor are parallel aligned. Otherwise the SEL is reduced due to a lateral averaging over several periods. A detailed description of the alignment and the integration of the components are given in [10]-[12]. Applying a voltage to the two electrodes actuates the integrated mirror. The parallel plates of the electrodes have a capacitance of approximately $4 \mathrm{pF}$. During operation we applied a voltage plus an offset voltage resulting in a displacement of the mirror of 3.8 and $33 \mu \mathrm{m}$.

We tested the system by using a red laser with the wavelength of $633 \mathrm{~nm}$. The optical output of the lasers was $307 \mu \mathrm{W}$. The "photocurrent versus mirror displacement" curves were mapped to "photocurrent versus time" curves accounting for nonlinearities of the mirror motion. Afterwards the signal was transformed from the time-domain to the frequency domain. The expected resolution of the spectrometer is defined by the following:

$$
\mathrm{FWHM}=\Delta \lambda=\frac{\lambda^{2}}{2 \cdot \Delta x} .
$$

The Fourier transform of a sensor signal generated by a single laser emitting light at $633 \mathrm{~nm}$ is shown in Fig. 7.

The full-width at half-maximum (FWHM) of around $6 \mathrm{~nm}$ is in good agreement with (7). 


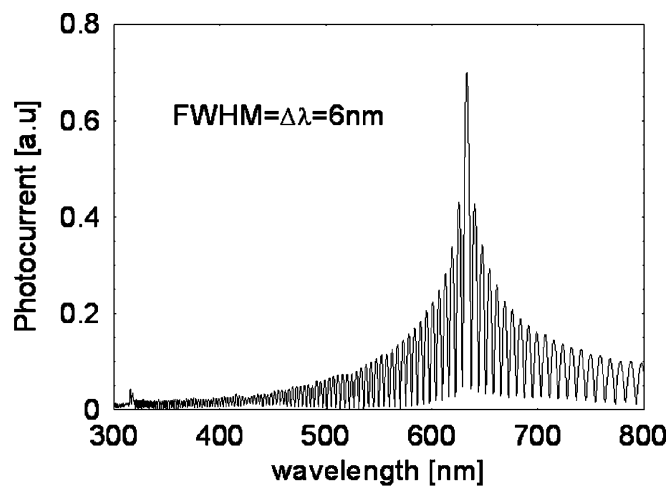

Fig. 7. Optical spectrum measured by an integrated Fourier spectrometer according to Fig. 2. A laser beam was used as an input signal. The micromachined mirror was actuated by $33 \mu \mathrm{m}$.

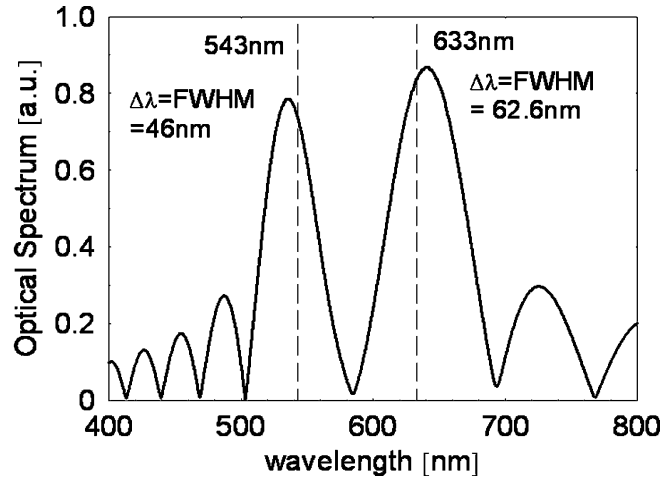

Fig. 8. Optical spectrum measured by an integrated Fourier spectrometer according to Fig. 2. Two laser beams (545 and $633 \mathrm{~nm}$ ) were used as an input signal. The micromachined mirror was actuated by $3.8 \mu \mathrm{m}$.

In the next step, we characterized the spectral discrimination and the linearity (concept of superposition of signals) of the spectrometer. We superimposed two laser beams with a wavelength of 543 and $633 \mathrm{~nm}$ and an optical output of 166 and $307 \mu \mathrm{W}$. After accounting for the position of the mirror the measured photocurrent spectra was transformed from the time domain to the frequency domain. The Fourier transform is shown in Fig. 8. In this case, the mirror was displaced by $3.8 \mu \mathrm{m}$. We determined an FWHM of 45 and $60 \mathrm{~nm}$ for the wavelengths of 543 and $633 \mathrm{~nm}$, which also exactly fits the predictions of (7). Fig. 8 exhibits that the spectral resolution of the spectrometer scales inversely with the scan length of the tunable mirror. Furthermore, the experimental data in Fig. 8 proves that the superposition principle can be applied.

\section{DISCUSSION}

A standing-wave spectrometer is a novel device structure, which facilitates the realization of Fourier spectrometer arrays. These arrays can open a new field of application where spectral and spatial sensor signals have to be fused. Other spectrometers like classical Fourier spectrometers or Grating spectrometers do not allow for a dense integration of hundreds or thousands of spectrometers on a single chip. So far imaging spectrometers have been realized by using a monochromator in front of a sensor array. However, such systems allow only for line scans because one dimension of the sensor array is used to capture the spectral information, whereas the other dimension is used to capture the spatial information.

In terms of the implementation, the Fabry-Perot spectrometer is closest to the novel standing-wave Fourier spectrometer. However, due to the different detection concepts Fabry-Perot spectrometers and standing-wave Fourier spectrometer exhibit different limitations concerning their qualities. The resolution of a Fabry-Perot spectrometer is limited by the absorption of the resonator and the reflectivity of the semi transparent mirrors. If an air resonator is formed the resolution is only limited by the reflectivity of mirrors. The resolution of the standing-wave spectrometer is limited by the scan length of the mirror.

Therefore, the standing-wave spectrometers combines the world of classical Fourier spectrometers with the world of Fabry-Perot spectrometers. The implementation of the standing-wave spectrometer is comparable with the realization of a Fabry-Perot spectrometer. In terms of the systems limitations the standing-wave spectrometer is comparable with a classical Fourier spectrometer.

The most important component of the novel Fourier spectrometer is the partly transparent optical detector. Such a detector can be realized as a coplanar or a sandwich structure. Throughout the paper we discussed so far the influence of the detector design on the overall performance of the standing-wave Fourier spectrometer. The sandwich structure (nip-photodiode) has the major advantage that the thin-film layer stack and the incoming optical light can be matched. The sandwich structure requires transparent conductive electrodes, which can be realized by ITO, $\mathrm{ZnO}$, or $\mathrm{SnO}_{2}$. The detector itself can than be realized as a photoconductor structure or a diode structure. In both cases the fabrication of a sandwich structure requires a high degree of process control. The processing of the ultra thin diode requires very smooth TCO-layers. In particular the preparation of the second TCO-layer is crucial. To avoid damage of the underlying amorphous layer the $\mathrm{TCO}_{\mathrm{II}}$-layer has to be sputtered at very soft conditions. A more detailed description of the deposition conditions is given in [15] and [18]. The fabrication of coplanar structures is simpler, because metal electrodes can be used to form the coplanar contacts.

Packaging, assembly, and alignment of the individual components of the integrated Fourier spectrometer are further important aspects, which have to be taken into account. The sandwich structure is beneficial with respect to the alignment of the beam and the individual components of the spectrometer. The sandwich structure is less sensitive to sensor misalignments. Due to a vertical device design the sensor is independent of the spot size of the incoming light beams. In the case of the coplanar structure the spot size has to be larger than the gap of the electrodes. Otherwise, the photoconductivity is limited by the position of the lowest illumination intensity. The coplanar detector design can be improved by using interdigitated contacts. On the other hand more light is reflected at the interdigitated metal contacts, so that an optimized design of the contacts is required. Further, light is diffracted by the interdigitated contacts, which affects the sensor signal.

Another important aspect of the thin film sensors are interface states, which negatively affect the sensor signal. This problem 
applies to both sensor concepts since both device structures are very thin. For such thin devices the electronic transport is influenced by interface states. Experimental results show that this mainly applies for coplanar structures. As a consequence the sensor exhibits nonlinearities with respect to the electric field dependence and the light intensity dependence of the photocurrent response.

The transient behavior of the sensor is another important parameter to characterize the partly transparent sensor. The geometric capacitance of the sandwich structure is high since the diodes are very thin and an $i$-layer thickness of only $40 \mathrm{~nm}$ was used. The resistance of the detector is mainly determined by the resistance of the TCO layers, which exhibit a resistivity of 25-62 $\Omega$ /square. Therefore, the $R C$ constant of the sensor leads to a limitation of the transient behavior. First experimental results exhibit cutoff frequencies in the range of $100-200 \mathrm{kHz}$.

The nip-diode can be combined with active matrix addressing to realize 1-D and 2-D arrays. An active matrix addressing is advantages because it facilitates the integration of pixel processing electronics. Advanced pixel electronics allows preprocessing of the sensor signal like data compression or filtering of the sensor signal. An example of an adaptive filter concept in combination with a standing-wave spectrometer is given in [20].

\section{SUMMARY}

The optical design of a novel spectrometer based on sampling a standing-wave was presented. The spectrometer consists of a partly transparent sensor and a tunable micromachined mirror.

Amorphous silicon nip-photodiodes were fabricated and integrated together with a micromachined mirror. Amorphous silicon is a perfect material for optoelectronic applications in the visible part of the spectrum, because the sensitivity matches excellent with the sensitivity of the human eye. The spectral resolution fits with the expectation so that the resolution inversely scales with the displacement of the mirror. The presented novel Fourier spectrometer reduces the number of components to a minimum, simplifies the setup to a linear arrangement of a detector and a mirror and facilitates the realization of spectrometer arrays. In terms of the resolution the novel standing-wave spectrometer is comparable with a classical Fourier spectrometer, whereas the realization of the device is closer to the realization a Fabry-Perot spectrometer.

\section{ACKNOWLEDGMENT}

The authors like to thank O. Kluth, Research Center Jülich, for many helpful discussions and technical support.

\section{REFERENCES}

[1] R. F. Wolffenbuttel, "State-of-the-art in integrated optical microspectrometers," IEEE Trans. Instrum. Meas., vol. 53, no. 1, p. 197, Feb. 2004.

[2] S.-H. Kong, J. H. Correia, G. De Graaf, M. Bartel, and R. F. Wolfenbuttel, "Integrated silicon microspectrometer," IEEE Instrum. Meas. Mag., p. 34, 2001.
[3] P. M. Zavracky, K. L. Denis, H. Xie, T. Wester, and P. Kelley, "Micromachined scanning Fabry-Perot interferometer," in Proc. SPIE, vol. 3514, 1998, pp. 179-187.

[4] C. Solf, C. Mohr, and U. Wallrabe, "Miniaturized LIGA Fourier transformation spectrometer," in Prof. Sensor Conf., Montreal, QC, Canada, 2003, p. 773.

[5] O. Manzardo, "Micro-sized Fourier spectrometers," Ph.D. dissertation, Univ. Neuchâtel, Switzerland, 2002.

[6] O. Manzardo, H. P. Herzig, C. R. Marxer, and N. F. de Rooij, "Miniaturized time-scanning Fourier transform spectrometer based on silicon technology," Opt. Lett., vol. 24, p. 1705, 1999.

[7] D. A. B. Miller, "Laser tuners and wavelength-sensitive detectors based on absorbers in standing-waves," IEEE J. Quantum Electron., vol. 30, no. 3, p. 732, Mar. 1994.

[8] H.-J. Buechner, German Patent: DE 3300 369, Jan., 071983.

[9] D. H. Alexander, K. Ishizuka, and R. N. Sato, U.S. Patent 4443 107, Apr. 17, 1984.

[10] H. L. Kung, S. R. Bhalotra, J. D. Mansell, and D. A. B. Miller, "Compact transform spectrometer based on sampling wave," in IEEE/LEOS Int. Optical MEMS Conf., Kauai, HI, 2000.

[11] H. L. Kung, S. R. Bhalotra, J. D. Mansell, D. A. B. Miller, and J. S Harris Jr., "Standing-wave transform spectrometer based on integrated MEMS mirror and thin-film photodetector," IEEE J. Sel. Top. Quantum Electron., vol. 8, no. 1, p. 98, Jan. 2002.

[12] S. R. Bhalotra, H. L. Kung, J. Fu, N. C. Helman, O. Levi, D. A. B. Miller and J. S. Harris Jr., "Integrated standing-wave transform spectrometer for near infrared optical analysis," in Proc. IEEE Lasers Electro-Optics Society Annual Meeting, Glasgow, U.K., 2002.

[13] H. Stiebig, H.-J. Büchner, E. Bunte, V. Mandryka, D. Knipp, and G Jäger, "Standing wave detection by thin transparent n-i-p diodes of amorphous silicon," Thin Solid Films, vol. 427, pp. 152-156, 2003.

[14] Z. Knittl, Optics of Thin Films. New York: Wiley, 1976.

[15] H. Stiebig, A. Kreisel, K. Winz, M. Meer, N. Schultz, T. Eickhoff, and $\mathrm{H}$. Wagner, "Spectral response modeling of a-Si:H solar cells using accurate light absorption profiles," in Proc. 1st World Conf. Photovoltaic Energy Conversion, 1994, p. 603.

[16] D. Knipp, P. G. Herzog, and H. Stiebig, "Stacked amorphous silicon color sensors," IEEE Trans. Electron Devices, vol. 49, no. 1, pp. 170-176, Jan. 2002.

[17] D. Knipp, M. Rosa, H. Stiebig, H.-J. Büchner, and R. A. Street, "Design and modeling of a Fourier spectrometer based on sampling a standingwave," Proc. Mater. Res. Soc., vol. 687, p. BS.39, 2001.

[18] O. Kluth, A. Löffl, S. Wieder, C. Beneking, L. Houben, B. Rech, H. Wagner, S. Waser, J. A. Selvan, and H. Keppner, "Texture etched Al-doped $\mathrm{ZnO}$ : a new material for enhanced light trapping in thin film solar cells," in Proc. IEEE PVSEC, 1997, pp. 715-718.

[19] W. Luft and Y. Tuso, Hydrogenated Amorphous Silicon Alloy Deposition Processes. New York: Marcel Dekker, 1993.

[20] S. R. Bhalotra, H. L. Kung, Y. Jiao, and D. A. B. Miller, "Adaptive timedomain filtering for real-time spectral discrimination," Opt. Lett., vol. 27, pp. 1147-1149, 2002.

[21] G. Jaeger and H.-J. Buechner, German Patent DE 3612 1221, Apr. 11 1986.

[22] A. Haiduk, A. Kottwitz, R. Legler, H.-J. Buechner, and K. Gerhardt, German Patent DE 4017 201, May, 29, 1990.

Dietmar Knipp (M'03) received the M.S. and Ph.D. degrees in electrical engineering from the University Siegen, Siegen, Germany, and the Aachen University of Technology, Aachen, Germany, in 1995 and 1999, respectively.

From 1996 to 2000, he was with the Research Center Jülich first as a Research Assistant, followed by Postdoctoral Scientist working on amorphous and nanocrystalline silicon and its application for optical sensors. From 2000 to 2002, he was with the Palo Alto Research Center Inc., Palo Alto, CA, where he carried out research on organic and molecular electronics. Until joining International University Bremen (IUB) as an Assistant Professor of Electrical Engineering in 2003, he was Senior Researcher at the Interuniversity Microelectronic Center (IMEC), Leuven, Belgium, working in the area of advanced components and sensor systems. His research focuses on electronic devices and photonics, with particular emphasis on organic and molecular transistors, nanophotonics, and optical sensor technologies. 
Helmut Stiebig received the Ph.D. degree in electrical engineering from the Aachen University of Technology, Aachen, Germany, in 1997.

Since 1992, he has been with the Research Center Jülich, Institute of Photovoltaics, Jülich, Germany. He is currently a head of the Device Analysis and Sensor Technology Research Group. His research interests include the investigation of the optoelectronic properties of amorphous and microcrystalline silicon and related materials, development of innovative device structures for solar cell applications, device analysis and numerical modeling, and the development of optical sensors based on thin-film technology.

Sameer R. Bhalotra ( $\mathrm{S}^{\prime}$ '01) received the A.B. degree in chemistry and physics from Harvard University, Cambridge, MA, in 1998 and the Ph.D. degree in Applied Physics from Stanford University, Stanford, CA, in 2004. His graduate research involved the development of miniaturized optical sensing systems.

Dr. Bhalotra was awarded the Regina Casper Stanford Graduate Fellowship in 1999, the Optical Society of America-Dekker Foundation Student Scholarship in 2003, and the IEEE Laser and Electro-Optics Society Graduate Fellowship in 2003 .

Eerke Bunte received the diploma and the Ph.D. degree in electrical engineering from the TU Braunschweig, Germany, in 2000 and 2004, respectively. his $\mathrm{Ph}$.D. research dealt with a photodetector for length measurements.

He has been with the Institute of Photovoltaics at the Research Center Jülich, Jülich, Germany, since 1999. His research interests include thin-film silicon solar cells and optical sensors for different applications.
Helen L. Kung (S'99) received the B.S. and Ph.D. degrees in electrical engineering from Brown University, Providence, RI, in 1996 and from Stanford University, Stanford, CA, in 2003, respectively. Her graduate research involved MEMS optical spectral sensors.

Since 2003, she has worked at Intel Corporation, Santa Clara, CA as a Senior Integration Engineer for Flash memory processes.

David A.B. Miller (M'84-SM'89-F'95) received the B.Sc. degree from St. Andrew's University, Fife, U.K., and the Ph.D. degree from Heriot-Watt University, U.K., in 1979, both in physics.

He was with Bell Laboratories from 1981 to 1996, and was made Department Head of the Advanced Photonics Research Department in 1987. He is currently the W. M. Keck Professor of Electrical Engineering at Stanford University, Stanford, CA, and the Director of the Ginzton and Solid State and Photonics Laboratories. His research interests include quantum-well optoelectronic and nanophotonic physics and devices, and fundamentals and applications of optics in information, sensing, switching, and processing. He has published more than 200 scientific papers, and holds over 55 patents.

Dr. Miller has served as a Board Member for both the Optical Society of America (OSA) and IEEE Lasers and Electro-Optics Society (LEOS), and in various other society and conference committees. He was President of the IEEE Lasers and Electro-Optics Society in 1995. He was awarded the Adolph Lomb Medal and the R. W. Wood Prize from the OSA, the International Prize in Optics from the International Commission for Optics, and the IEEE Third Millennium Medal. He is a Fellow of the Royal Societies of London and Edinburgh, OSA, and the APS, and holds honorary degrees from the Vrije Universiteit Brussel and Heriot-Watt universities. 